\title{
Factors affecting difficult lightwand intubation: a prospective double-blind trial
}

\author{
Department of Anesthesia and Pain Medicine, Ilsan Hospital, Dongguk University College of Medicine, Goyang, \\ *Department of Anesthesia and Pain Medicine, Sanggye Paik Hospital, Inje University College of Medicine, Seoul, Korea
}

\author{
Younsuk Lee, Sangseok Lee*, Jun Heum Yon*, and Jeoung Hyuk Lee
}

\begin{abstract}
Background: Unanticipated difficulties during tracheal intubation are related to perioperative morbidity and mortality, but the success of direct laryngoscopic intubation depends largely on clinician's experience and the upper airway anatomy. The lightwand was introduced as alternative intubation technique, but the indicators of difficult lightwand intubation (DLWI) have not been identified. Accordingly authors conducted this study to identify subject factors that affect DLWI, and to compare these with those of difficult laryngoscopic intubation.

Methods: Seventy-three healthy subjects requiring tracheal intubation for elective surgery were enrolled. Anatomic factors, such as, body mass index (BMI), Mallampati classification (MC), inter-incisor gap, thyromental distance, neck circumference, extent of head and neck motion, and Cormack-Lehane grade (CL) were determined and evaluated in terms of their abilities to predict DLWI, which was described using intubation time and number of intubation attempts. Multiple regression analyses were performed to identify predictors using a variable selection technique.

Results: Only MC and BMI were found to predict DLWI. The weighted sum of time and number of attempts $\left(r^{2}=0.854\right.$, $\mathrm{P}=0.000)$ was found to be better predictor of DLWI than their product $\left(\mathrm{r}^{2}=0.734, \mathrm{P}=0.000\right)$. Cormack-Lehane grade was not found to be significantly related to DLWI $(\mathrm{P}=0.093)$.

Conclusions: Of the anatomic factors examined, only Mallampati classification and body mass index were found to predict difficult lightwand intubation. (Korean J Anesthesiol 2009; 56: 18 24)
\end{abstract}

Key Words: Body mass index, Cormack grade, Difficult intubation, Endotracheal intubation, Mallampati class.

\section{INTRODUCTION}

Lightwands are now used for endotracheal intubation as an alternative to direct laryngoscopic intubation in Korea. Furthermore prospective $^{1-6)}$ and retrospective ${ }^{7)}$ studies have shown that certain subject-associated factors predict difficult laryngoscopic intubation. However, reported predictors of difficult lightwand intubation (DLWI) are inconsistent, and furthermore, it has not been established whether predictors of difficult laryngoscopic intubation also predict DLWI. According to

Received: August 21, 2008.

Accepted: November 1, 2008.

Corresponding author: Younsuk Lee, M.D., Department of Anesthesia and Pain Medicine, Ilsan Hospital, Dongguk University College of Medicine, 814, Siksa-dong, Ilsan-gu, Goyang 411-773, Korea. Tel: 82-31-961-7872, Fax: 82-31-961-7864, E-mail: ylee@dongguk.ac.kr

ESA Congress, 2008, Denmark.

Copyright (C) Korean Society of Anesthesiologists, 2009
Inoue, ${ }^{8)}$ lightwand intubation success rates are comparable with those of laryngoscopic intubation. Moreover, difficult laryngoscopic intubation can be relieved using a lightwand, ${ }^{9,10)}$ and DLWI can be relieved using a laryngoscope. ${ }^{10)}$

Endotracheal intubation is the mainstay of current anesthetic practice, and the prediction of difficult intubation is major concern for anesthetists. Furthermore, unanticipated difficult intubation is associated with morbidity and mortality during general anesthesia. Accordingly, the authors conducted this study to identify the subject-associated factors that affect DLWI, and to compare these factors with those that predict difficult laryngoscopic intubation (DLSI).

\section{MATERIALS AND METHODS}

After obtaining institutional research board approval and written informed consent, 73 ASA PS Class I-III adult subjects scheduled to undergo a variety of surgical procedures requiring 
endotracheal intubation were enrolled in this study. The following preanesthetic measurements were recorded; body weight, height, body mass index (BMI), graded visualization of the pharynx using the Mallampati classification (MC), inter-incisor gap (IIG), thyromental distance (TMD), neck circumference (NC) at the level of the cricoid, and extent of head and neck motion (HNM) (Table 1).

Glycopyrrolate, $0.2 \mathrm{mg}$ was given intravenously 30 minutes prior to arrival in the operating room. Denitrogenation was instituted under conventional monitoring. After achieving anesthetic induction with a titrated dose of propofol and verifying the adequacy of mask ventilation, neuromuscular blocking drugs and volatile anesthetics were administered as required. Mask ventilation was continued with an oxygen-volatile anesthetic mixture for 3-4 minutes. Direct laryngoscopy was performed to grade larynx visualization according to the Cormack-Lehane (CL) classification. ${ }^{11)}$ After reinstalling mask ventilation for a few minutes, intubation was attempted by an anesthetist unaware of CL grade using a lightwand. The lightwand procedures used were as described in our institution's local standard anesthetic guideline. Briefly, the tip of the lightwand (Surch-Lite ${ }^{\mathrm{TM}}$, AARON Medical, St. Petersburg, USA) was bent at about 90 degrees near the proximity of its cuff, the subject's mandible was then elevated with a thumb and index finger, and the operating room light was dimmed, a midline transilluminating glow over the thyroid cartilage was deemed to indicate optimal positioning of the tube-lightwand couplet in the larynx, and a cuffed ID (internal diameter) 7.0 or $7.5 \mathrm{~mm}$ endotracheal tube was then advanced over the lightwand into the trachea. Intratracheal placement of the endo-

Table 1. Physical Measurements and Number of Attempts according to Mallampati Classification

\begin{tabular}{lcccc}
\hline & & \multicolumn{3}{c}{ Mallampati classification } \\
\cline { 3 - 5 } & Overall & 1 & 2 & 3 \\
\hline $\mathrm{N}$ & & 25 & 30 & 18 \\
Sex (M/F) & $35 / 38$ & $12 / 13$ & $16 / 14$ & $7 / 11$ \\
Age (yrs) & $44 \pm 15$ & $41 \pm 14$ & $42 \pm 14$ & $51 \pm 14$ \\
Body weight (kg) & $70 \pm 16$ & $67 \pm 16$ & $74 \pm 14$ & $66 \pm 18$ \\
Height (cm) & $165 \pm 10$ & $165 \pm 14$ & $166 \pm 7$ & $162 \pm 9$ \\
Number of & $58 / 9 / 6$ & $21 / 2 / 2$ & $23 / 5 / 2$ & $14 / 2 / 2$ \\
attempts (1/2/3) & & & & \\
\hline
\end{tabular}

Data is presented as means \pm SD except for sex data and number of attempts. Data shows no statistical significance according to Mallampati classification. tracheal tube was confirmed by auscultation of bilateral breathing sounds and by using expired $\mathrm{CO}_{2}$ waves. When the endotracheal tube was not in the trachea, it was withdrawn and intubation was re-attempted by the same anesthetist until the correct endotracheal tube positioning was confirmed.

Intubation time (defined as the time from mandible elevation to complete unthreading of the lightwand) was recorded as were the number of attempts made. Times required for preceding unsuccessful attempts were ignored, only the times required for the final successful attempts were recorded. Degree of difficulty was determined based on the time required for final successful insertions.

\section{Data selection and analyses}

In terms of the two indices used to quantify degrees of DLWI, the product of intubation time and number of attempts (time-attempt product) was intuitive, and the weighted sum of time and attempts (weighted sum) was more explanatory (Appendix). Anatomic factors including BMI $\left(\mathrm{kg} / \mathrm{m}^{2}\right), \mathrm{MC}(1-3)$, IIG $(\mathrm{cm})$, TMD (cm), NC (cm) and HNM (1/0) were investigated as potential predictors of DLWI.

Initially, correlation analyses were performed to assess associations between each factor and the time-attempt product. Correlations were described using Pearson's product-moment coefficient for BMI, IIG, TMD and NC, or using $\tau$ (Kendall's tau) for MC and HNM. Significance was determined using $\mathrm{P}$ values. Second, we searched for correlations between MC and the other factors, and those found to be correlated with MC were excluded to eliminate bias due to collinearity. Third, to identify fits between time-attempt product and candidate factors not correlated with MC, we used stepwise selection method. Fourth, only those factors selected during the previous step were investigated to identify fits between weighted sums and candidate factors. Finally, CL was arbitrarily added to the equation to investigate its additional influence on DLWI. Models containing and not containing CL were compared by analysis of variance.

Of the factors examined, $\mathrm{MC}$ and $\mathrm{CL}$ were treated as ordered orthogonal polynomial contrasts, and HNM as the Helmert contrasts. Statistical analyses were performed using S-Plus 8.0 (Insightful Corp., USA), matrix operations using GNU Octave 2.1.73 (John W. Eaton, USA) and statistical power calculations using Power and Precision 3.2 (Biostat, USA). Statistical significance was accepted for $\mathrm{P}$ values of $<$ 0.05 . 


\section{RESULTS}

Intubation time averaged $18 \pm 8 \mathrm{sec}$, and in 58 subjects, tracheas were intubated at first attempts. Number of attempts was not significantly different for different $\mathrm{MC}$ classes $(\mathrm{P}=$ 0.576). There was no failed intubation.

When assessing the influence of individual factors, significant correlations were found between the time-attempt product and MC and BMI (Table 2). Because NC, HNM, and CL were found to be significantly correlated with $\mathrm{MC}$ (Fig. 1), BMI, IIG, and TMD were included with MC in the third step. Only MC and BMI were selected as predictors and fitted with time-attempt product values $\left(\mathrm{r}^{2}=0.734, \mathrm{P}=0.000\right)$.

$$
\begin{aligned}
& \text { Time-Attempt Product }=\mathrm{M}_{0}+0.669 \times \text { BMI (Equation 1) } \\
& \left(\mathrm{M}_{0}=-2.880,6.603 \text { and } 8.733\right. \text { for Mallampati classi- } \\
& \text { fication 1, } 2 \text { and 3) }
\end{aligned}
$$

Canonical weighs, referred to as w1 and w2 were 0.999955036 and -0.009482981 for time and for number of attempts by canonical correlation analysis (Appendix). Weighted sums were calculated using w1 time + w2 $\cdot$ attempt. Described with weighted sum, the degrees of DLWI were rather tightly fit on MC and BMI than those with time-attempt product was:

Weighted Sum $(0.999955036 \cdot$ time $-0.009482981 \cdot$ at-

Table 2. Solitary Influence of Anatomic Factors on the Time-Attempt Product

\begin{tabular}{lcc}
\hline & Correlation $^{\ddagger}$ & Significance \\
\hline Body mass index (BMI, kg/m ${ }^{2}$ ) & 0.243 & 0.043 \\
Mallampati classification (MC, 1-3*) & 0.218 & 0.003 \\
Inter-incisor gap (IIG, cm) & -0.069 & 0.704 \\
Thyromental distance (TMD, cm) & -0.199 & 0.128 \\
Neck circumference (NC, cm) & 0.151 & 0.365 \\
Head and neck motion (HNM, 1/0 ${ }^{\dagger}$ ) & 0.108 & 0.290 \\
\hline
\end{tabular}

* $\mathrm{MC}$ is coded using ordered orthogonal polynomial contrasts combining linear (.L) and quadratic (.Q) components. Corresponding contrast matrix is described by:

\begin{tabular}{lrr} 
& \multicolumn{1}{c}{. $\mathrm{L}$} & \multicolumn{1}{c}{. $\mathrm{Q}$} \\
MC 1 \\
MC 2 \\
MC 3
\end{tabular}$\quad\left[\begin{array}{rr}-0.7071068 & 0.4082483 \\
0.0000000 & -0.8164966 \\
0.7071068 & 0.4082483\end{array}\right]$

${ }^{\dagger} \mathrm{HNM}$ is coded 1 when immobile, 0 mobile. ${ }^{\ddagger}$ Correlations were estimated using Pearson's product-moment coefficient, except MC and HNM, which were estimated using Kendall's tau. tempts) $=\mathrm{M}_{0}+0.510 \times$ BMI (Equation 2)

$\left(\mathrm{M}_{0}=2.300,5.285\right.$ and 7.609 for Mallampati classifications 1,2 and 3) $\left(\mathrm{r}^{2}=0.854, \mathrm{P}=0.000\right)$ (Fig. 2)

Adding $\mathrm{CL}$ to Equation 2 did not alter Equation 2 significantly $\left(\mathrm{r}^{2}=0.868, \mathrm{P}=0.093\right)$. Power for an $\mathrm{r}^{2}$ increment of 0.014 from 0.854 to 0.868 was estimated at 1.00 .

\section{DISCUSSION}

The reader should be aware that in the present study we did not dichotomize degrees of DLWI or predictors, such as, MC $\geq 3,{ }^{12-14)} \mathrm{TMD} \leq 6.5$ or $7 \mathrm{~cm},{ }^{12,14)} \mathrm{IIG} \leq 4 \mathrm{~cm},{ }^{14)}$ or their scoring systems. ${ }^{15,16)}$ Instead, we calculated the canonically weighted sum of intubation time and number of attempts, which provide the correlations between degrees of DLWI and the predictors. In addition, we excluded CL from the regression model to predict DLWI a priori and a posteriori.

Researchers tend to regard difficult intubation as a binary state or as a sum of several binary states, i.e., subjective feeling, ${ }^{2,17,18)} \mathrm{CL} \geq 3,{ }^{19,20)}$ and the need for external laryngeal pressure. ${ }^{1)}$ When described using binary states, difficult intubation can be easily understood, but such descriptions are oversimplification. Because difficult intubation is a subjective issue, we attempted to grade intubation difficulties in ways that were compatible with clinical situations. In the present study, DLWI was defined to be complex function incorporating intubation time and number of attempts. In addition, we also graded all study factors, except for limited head and neck motion. Furthermore, we adopted the assertion by Royston and colleagues $^{21)}$ that although dichotomized predictors are straightforward, they introduce the risk of a considerable loss of power and residual confounding.

Our analysis is unique because the determined DLWI index is not simple product of intubation time and number of attempts. The inclusion of number of intubation attempts we believed minimizes errors associated with describing intubation difficulty in terms of intubation time alone, although it is debatable whether failure during an initial attempt constitutes a measure of difficulty. Moreover, we considered that the intubation time/number of attempts product exaggerated DLWI, and thus, we examined whether a DLWI was better described using a weighted sum. In the event our findings indicate that this enhances the strengths of correlations between DLWI and the predictors. The negative weighting for number of attempts 

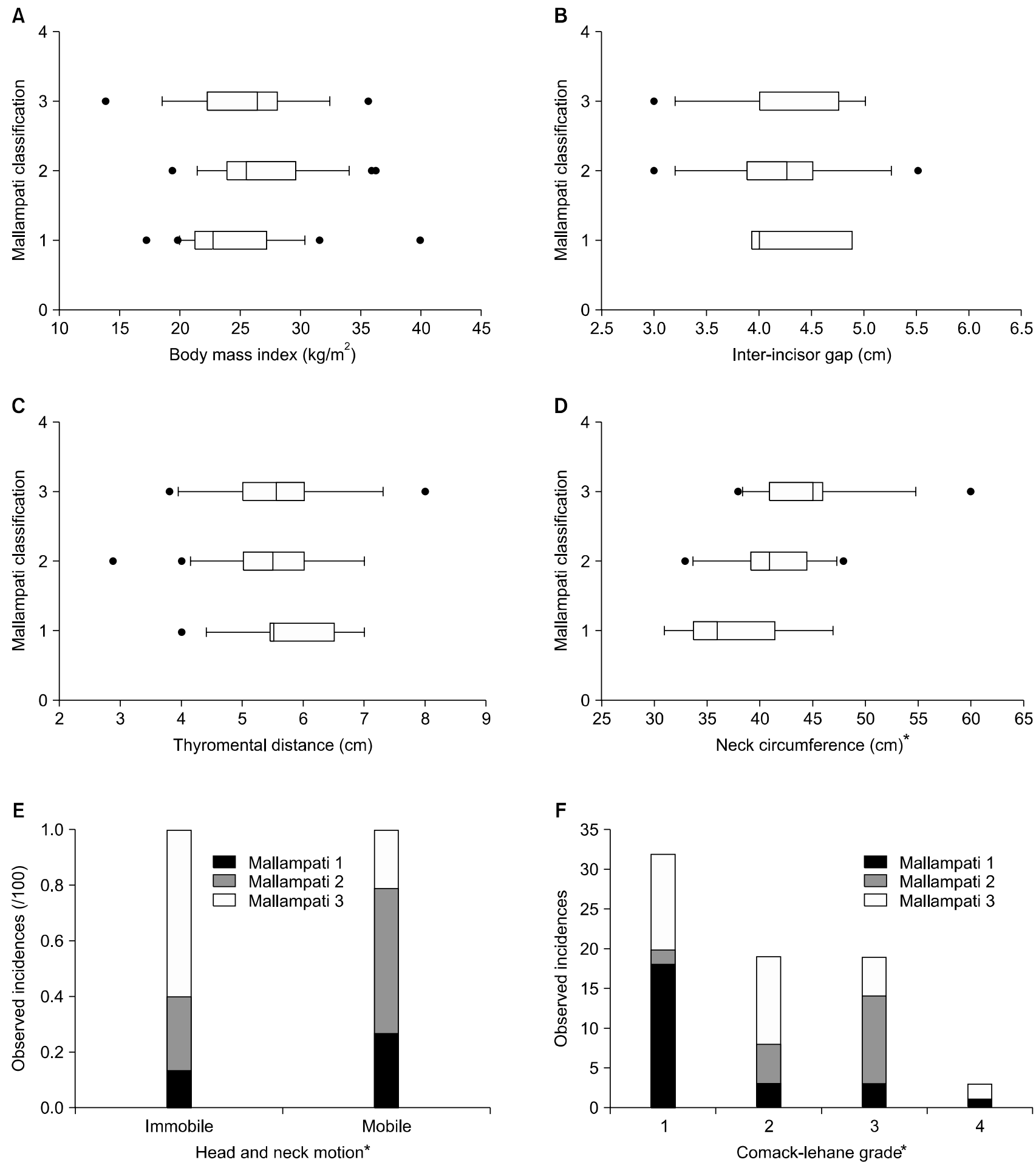

Fig. 1. Correlation between Mallampati classification (MC) and subject factors. (A) body mass index (BMI, $\tau=0.128, P=0.094)$, (B) inter-incisor gap (IIG, $\tau=0.021, \mathrm{P}=0.848),(\mathrm{C})$ thyromental distance $(\mathrm{TMD}, \tau=-0.059, \mathrm{P}=0.470)$, (D) neck circumference $(\mathrm{NC}, \tau$ $=0.313, \mathrm{P}=0.003)$, (E) limited head and neck motion $(\mathrm{HNM}, \tau=0.198, \mathrm{P}=0.039)$, $(\mathrm{F})$ Cormack-Lehane grade $(\mathrm{CL}, \tau=0.272, \mathrm{P}=$ 0.000). Factors correlated significantly with $\mathrm{MC}, \mathrm{NC}, \mathrm{HNM}$ and $\mathrm{CL}$ were deselected to predict the difficulty of lightwand incubation (DLWI). The letter $\tau$ denotes Kendall's tau, which indicates the correlation between the variable concerned and Mallampati classification. Asterisk $(*)$ denotes statistical significance $(\mathrm{P}<0.05)$. 


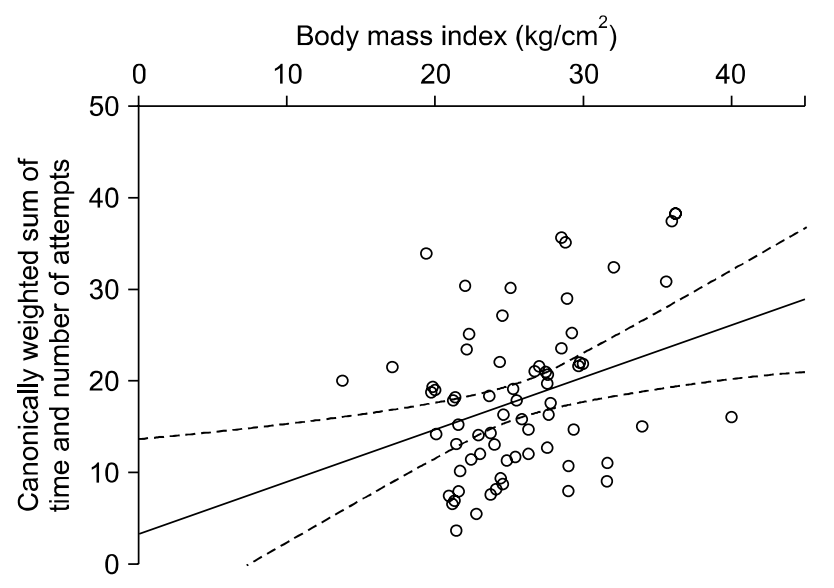

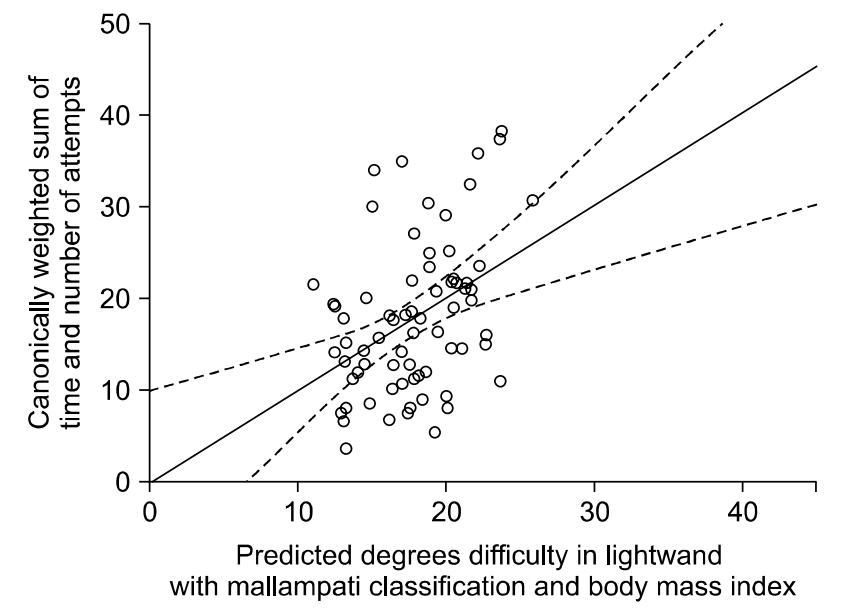

appears to be counterintuitive, but it provides an interesting interpretation of data, as it indicates that repeated attempts prolonged the elapsed time beyond the virtual influence of repetition on degrees of DLWI, and that repetition was usually not caused by true difficulty but by an uncomplicated lapse. The lack of a significant correlation between number of attempts and Mallampati classification is also in-line with this negative weighting. Aware of the mathematical assumption that variances of canonical correlation analysis are not normally distributed, our results might not be generalized, and one should recalculate the weights from their own data, for our weights belong only with our data.

CL grade, is related to observation of the larynx by an external observer, and appears to be valid in terms of assessing DLSI. However, in the case of the lightwand, the external view is no longer useful, because the endotracheal tube can be advanced irrespective of visualizing the larynx. ${ }^{22)}$ It is important to provide sufficient pharyngeal space to rotate and to advance the lightwand-tube couplet during lightwand intubation.

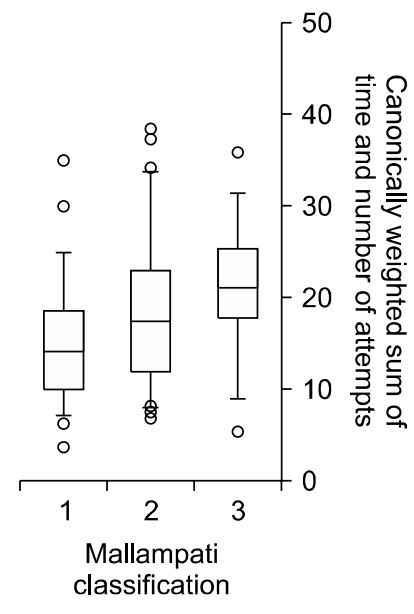

Fig. 2. Weighted sum of time and number of attempts versus Mallampati classification $\left(r^{2}=0.845, P=\right.$ 0.000 , right lower plane), body mass index $\left(r^{2}=0.842, P=0.000\right.$, left upper plane), and their combination $\left(r^{2}=0.854, P=0.000\right.$, left lower plane). Difficult lightwand intubation (DLWI) as described by the weighted sum was found to be well explained using a combination of Mallampati classification and body mass index
Our final equation includes only MC and BMI as predictors of DLWI, which suggests that the size and shape of the pharyngeal space substantially explains DLWI, as MC reflects the size of the pharyngeal space, and BMI reflects its size and shape, as was reported by Mayer and colleagues. ${ }^{23)}$

Physicians always encounter DLWI in ignorance of CL, and although CL is expected to be ignored when predicting DLWI, we included $\mathrm{CL}$ in the final equation to determine the additional influence of CL on DLWI, and the relation between DLWI and DLSI. The additional influence of CL in terms of predicting DLWI was weak, and a low MC, even with a high $\mathrm{CL}$, allows easy lightwand insertion, whereas a low CL with a high MC allows easy laryngoscopic insertion, which supports anecdotal evidence and the findings of previous reports, i.e., that a lightwand can relieve DLSI ${ }^{9,10)}$ and a laryngoscope can relieve DLWI vice versa. ${ }^{10)}$ However, weak influence of $\mathrm{CL}$ on DLWI was be extracted. Thus, the weak additional influence of CL on DLWI should be interpreted carefully, due to the probable collinearity between CL and MC (Fig. 1(F)). 
A previous report by Wong and colleagues ${ }^{18)}$ partly agrees with our result concerning the exclusion of CL, i.e., that MC and not $\mathrm{CL}$ influences intubation time. However, we disagree with their method of analysis, because Kruskal-Wallis analysis, (if the ordered characteristics of MC and CL are ignored,) always causes a loss of statistical power. Because we are unable to calculate the statistical power post hoc of the negative result of Wong and colleagues, ${ }^{18)}$ direct comparison might be invalid. Our result on the influence of CL is novel in that the power value of the present study reached 1.00 when CL was excluded from the set of predictors. When we treated $\mathrm{MC}$ and $\mathrm{CL}$ as ordered factors, we found that CL did not additionally influence DLWI. The present study demonstrates that lightwand intubation difficulty is well explained by the Mallampati classification and body mass index, which means that the size and shape of pharyngeal space most dictate lightwand intubation difficulty.

\section{ACKNOWLEDGEMENT}

2007 Research Fund of Inje University 2008 Research Fund of Dongguk University.

\section{REFERENCES}

1. Adnet F, Borron SW, Racine SX, Clemessy JL, Fournier JL, Plaisance $P$, et al: The intubation difficulty scale (IDS): proposal and evaluation of a new score characterizing the complexity of endotracheal intubation. Anesthesiology 1997; 87: 1290-7.

2. Arné J, Descoins P, Fusciardi J, Ingrand P, Ferrier B, Boudigues $D$, et al: Preoperative assessment for difficult intubation in general and ENT surgery: predictive value of a clinical multivariate risk index. Br J Anaesth 1998; 80: 140-6.

3. Ayuso MA, Sala X, Luis M, Carbó JM: Predicting difficult orotracheal intubation in pharyngo-laryngeal disease: preliminary results of a composite index. Can J Anaesth 2003; 50: 81-5.

4. Charters P: Analysis of mathematical model for osseous factors in difficult intubation. Can J Anaesth 1994; 41: 594-602.

5. Egan TD, Wong KC: Predicting difficult laryngoscopy for tracheal intubation: an approach to airway assessment. Ma Zui Xue Za Zhi 1993; 31: 165-78.

6. Gonzalez H, Minville V, Delanoue K, Mazerolles M, Concina D, Fourcade O: The importance of increased neck circumference to intubation difficulties in obese patients. Anesth Analg 2008; 106: 1132-6.

7. Fahy L, Horton WA, Charters P: Factor analysis in patients with a history of failed tracheal intubation during pregnancy. $\mathrm{Br} \mathrm{J}$
Anaesth 1990; 65: 813-5.

8. Inoue Y: Lightwand intubation can improve airway management. Can J Anaesth 2004; 51: 1052-3.

9. Agrò F, Totonelli A, Gherardi S: Planned lightwand intubation in a patient with a known difficult airway. Can J Anaesth 2004; 51: 1051-2.

10. Hung OR, Pytka S, Morris I, Murphy M, Launcelott G, Stevens $\mathrm{S}$, et al: Clinical trial of a new lightwand device (Trachlight) to intubate the trachea. Anesthesiology 1995; 83: 509-14.

11. Cormack RS, Lehane J: Difficult tracheal intubation in obstetrics. Anaesthesia 1984; 39: 1105-11.

12. Tse JC, Rimm EB, Hussain A: Predicting difficult endotracheal intubation in surgical patients scheduled for general anesthesia: a prospective blind study. Anesth Analg 1995; 81: 254-8.

13. Frerk CM: Predicting difficult intubation. Anaesthesia 1991; 46: 1005-8.

14. Merah NA, Wong DT, Ffoulkes-Crabbe DJ, Kushimo OT, Bode CO: Modified Mallampati test, thyromental distance and inter-incisor gap are the best predictors of difficult laryngoscopy in West Africans. Can J Anaesth 2005; 52: 291-6.

15. Wilson ME, Spiegelhalter D, Robertson JA, Lesser P: Predicting difficult intubation. Br J Anaesth 1988; 61: 211-6.

16. Nath G, Sekar M: Predicting difficult intubation--a comprehensive scoring system. Anaesth Intensive Care 1997; 25: 482-6.

17. Paix AD, Williamson JA, Runciman WB: Crisis management during anaesthesia: difficult intubation. Qual Saf Health Care 2005; 14: e5.

18. Wong SY, Coskunfirat ND, Hee HI, Li JY, Chen C, Tseng CH: Factors influencing time of intubation with a lightwand device in patients without known airway abnormality. J Clin Anesth 2004; 16: $326-31$.

19. Khan ZH, Gharabaghian M, Nilli F, Ghiamat M, Mohammadi M: Easy endotracheal intubation of a patient suffering from both Cushing's and Nelson's syndromes predicted by the upper lip bite test despite a Mallampati Class 4 airway. Anesth Analg 2007; 105: 786-7.

20. Kim JA, Lee JJ: Preoperative predictors of difficult intubation in patients with obstructive sleep apnea syndrome. Can J Anaesth 2006; 53: 393-7.

21. Royston P, Altman DG, Sauerbrei W: Dichotomizing continuous predictors in multiple regression: a bad idea. Stat Med 2006; 25 : 127-41.

22. Ainsworth QP, Howells TH: Transilluminated tracheal intubation. Br J Anaesth 1989; 62: 494-7.

23. Mayer P, Pépin JL, Bettega G, Veale D, Ferretti G, Deschaux C, et al: Relationship between body mass index, age and upper airway measurements in snorers and sleep apnoea patients. Eur Respir J 1996; 9: 1801-9.

24. Everitt B: An R and S-PLUS Companion to Multivariate Analysis. London, Springer-Verlag. 2007, pp 160-70. 


\section{$<$ APPENDIX $>$}

\section{Procedures}

In the present study, we adopted the canonical correlation procedures suggested by Everitt ${ }^{24)}$ to characterize the independent relationships that exist between sets of dependent variables. The theoretical equation was postulated as follows;

$\mathrm{w} 1 \cdot$ time $+\mathrm{w} 2 \cdot$ attempt $=$ sets of predictors (here, MC and BMI)

MC and BMI were selected from Equation 1. Correlation matrices were constructed for time, number of attempts, MC and BMI. Series of matrices, $r_{12}, r_{22}, r_{12}$ and $r_{21}$ were defined as follows;

Correlation matrices were arrayed among time, number of attempts, MC and BMI. Series of matrices, $r_{12}, r_{22}, r_{12}$ and $r_{21}$ were defined as follows;

$$
\begin{aligned}
& \mathrm{r}_{11}=\left(\begin{array}{cc}
1.0 & \mathrm{r}_{\text {attempt, time }} \\
\mathrm{r}_{\text {time, attempt }} & 1.0
\end{array}\right) \\
& \mathrm{r}_{22}=\left(\begin{array}{cc}
1.0 & \mathrm{r}_{\mathrm{BMI}, \mathrm{MC}} \\
\mathrm{r}_{\mathrm{MC}, \mathrm{BMI}} & 1.0
\end{array}\right) \\
& \mathrm{r}_{12}=\left(\begin{array}{cc}
\mathrm{r}_{\mathrm{MC}, \text { time }} & \mathrm{r}_{\mathrm{BMI}, \text { time }} \\
\mathrm{r}_{\mathrm{MC}, \text { attempt }} & \mathrm{r}_{\text {time,MC }}
\end{array}\right) \\
& \mathrm{r}_{21}=\mathrm{r}_{12}{ }^{\mathrm{T}}
\end{aligned}
$$

Matrix $\mathrm{E}$ is the solution of a calculation based on the least-square principle. The symbol $\mathrm{r}_{\mathrm{A}, \mathrm{B}}$ represents the correlation coefficient between variables $A$ and $B$. Superscript $T$ represents matrix transposition, and superscript -1 the inverse matrix.

$$
E=r_{11}^{-1} \cdot r_{12} \cdot r_{22}^{-1} \cdot r_{21}
$$

To find a matrix $\mathrm{X}$ satisfying $\mathrm{E} \cdot \mathrm{X}=\lambda \cdot \mathrm{X}$ for any scalar $\lambda$, the eigenvector of matrix $\mathrm{X}$ was obtained. Weights, w1 and w2 were read in the first column, which represents eigenvectors, of matrix $\mathrm{X}$.

\section{Solutions}

Correlation matrices were defined as;

$$
\begin{aligned}
& r_{11}=\left(\begin{array}{cc}
1.0 & 0.1039205 \\
0.1039205 & 1.0
\end{array}\right) \\
& r_{22}=\left(\begin{array}{cc}
1.0 & 0.1275362 \\
0.1275362 & 1.0
\end{array}\right) \\
& r_{12}=\left(\begin{array}{cc}
0.2092846 & 0.3246769 \\
0.02929985 & 0.02533489
\end{array}\right) \\
& r_{21}=r_{12}{ }^{T}=\left(\begin{array}{cc}
0.2092846 & 0.3246769 \\
0.02929985 & 0.02533489
\end{array}\right)^{T}=\left(\begin{array}{ll}
0.2092846 & 0.02929985 \\
0.3246769 & 0.02533489
\end{array}\right)
\end{aligned}
$$

Matrix E was found;

$$
\begin{aligned}
& E=r_{11}{ }^{-1} \cdot r_{12} \cdot r_{22}{ }^{-1} \cdot r_{21} \\
& =\left(\begin{array}{cc}
0.134195723 & 0.01267270921 \\
-0.001271344 & 0.00001571773
\end{array}\right)
\end{aligned}
$$

Its eigenvector was obtained;

$$
\text { eigenvector }=\left(\begin{array}{cc}
0.999955036 & -0.09461933 \\
-0.009482981 & 1.00094228
\end{array}\right)
$$

Finally, w1 and w2 values were found to be 0.999955036 and w2 -0.009482981 , respectively. The second index of degree of DLWI was set to $0.999955036 \cdot$ time -0.009482981 • attempts. 